\title{
Evidence in Favour of IMF Variations
}

\author{
Frank Eisenhauer \\ Max-Planck-Institut für extraterrestrische Physik, \\ Giessenbachstrasse, 85741 Garching, Germany
}

\begin{abstract}
The stellar initial mass function (IMF) determines the relative number of stars born at a given mass. Despite the tremendous effort to establish a universal IMF, the astronomical literature offers a wealth of diverse evidence for IMF variations. This review was prepared for a controversial debate at the conference "Starbursts - Near and Far" at Ringberg Castle, 2000, and gives a one-sided portrayal in favour of IMF variations. I will summarise the empirical evidence that the IMF varies with time, with environment, and for all stellar masses. While I see no obvious systematic trend in most regions of our Galaxy, there is at least an indication that the IMF is biased towards more massive stars in the early universe and in starbursts.
\end{abstract}

\section{Introduction}

The masses of stars span at least 3 orders of magnitude, from approximately 100 solar masses for the highest mass stars to the hydrogen burning limit of 0.1 solar masses. The relative numbers of stars born at a given mass is described by the initial mass function (IMF). This distribution is extremely important for many fields in astronomy, from the theory of star formation to the interpretation of integrated properties of galaxies at the highest redshift. Although many investigators have measured the IMF in a variety of star forming regions, we have not yet been able to come up with a final conclusion about the universality of the IMF. Specifically, Scalo's article [38 for the recent conference "The Stellar Initial Mass Function" [7] has revived the discussion, and many scientists including the author - have not decided on their final opinion. Nevertheless the conference organisers have chosen to split the review on the universality of the IMF to keep the discussion lively and controversial. This contribution focuses strictly on the empirical evidence in favour of IMF variations. I will not try to balance any arguments, but leave the critical comparison with the evidence in favour of a universal IMF [8] to the reader.

The postulation of a universal IMF - in its strongest form - states that the IMF is and has always been the same in all regions of star formation everywhere; the frequencies of initial stellar masses in any unbiased sample are always drawn from the same statistical distribution. I will present evidence against this postulation with respect to "is and has always been" as well as to "everywhere", and I will also argue against this postulation being valid even in star forming regions of our own Galaxy.

Although challenging the concept of a universal IMF, I refer to a reference IMF for comparison. This IMF (small inset in figure 1) is the field star IMF 
defined by Scalo [37 in 1986, because its qualitative behaviour seems to be typical in many respects: (1) For high stellar masses the IMF can be described by a power law $\frac{d N}{d \log M} \propto M^{\Gamma}$. The exponent is referred to as slope of the IMF. In the notation of this article the value for the Salpeter 35] IMF is -1.35. This power law results from scale free star formation processes, for example dominated by turbulent pressure. (2) The IMF is flat - the slope of the power law approximately 0 - for masses around $0.5 M_{\odot}$, indicating a characteristic stellar mass, for example the thermal Jeans mass. (3) The IMF declines for low stellar masses and may be described by a log-normal distribution, indicating a large number of independent parameters in the star formation process. However, I will present evidence that the IMF differs in various regions from this reference IMF in shape (e.g. log-normal versus power law and turnover versus continuous), in slope (e.g. bias towards high or low masses) and in characteristic mass.

\section{The Early Universe}

If the IMF depends on any environmental conditions, one would expect the largest deviations for the most extreme environments. The early Universe is such an environment. There are two very compelling indications that the IMF was different in the early Universe: First, no metal-free stars and only few verylow-metallicity stars have ever been found [1],3], although low-mass stars, which have formed in the metal-poor early Universe, should have survived the last 15 billion years. Only $\approx 500$ stars are known with $[\mathrm{Fe} / \mathrm{H}] \approx \frac{1}{300}[\mathrm{Fe} / \mathrm{H}]_{\odot}-$ the lowest metallicity observed in Galactic globular clusters - , and only $\approx 100$ stars with $[\mathrm{Fe} / \mathrm{H}] \approx \frac{1}{1000}[\mathrm{Fe} / \mathrm{H}]_{\odot}$. No single star with primordial metallicity $[\mathrm{Fe} / \mathrm{H}]$ $\approx \frac{1}{1000000}[\mathrm{Fe} / \mathrm{H}]_{\odot}$ has ever been observed. Second, the solar neighbourhood is deficient in metal-poor stars [20]. This discrepancy with the field star IMF is known as the "classical G-dwarf problem". Because the lifetime of these stars is longer than the age of the Universe, we would expect to find many more such stars, if low-mass stars have formed with the same frequency in the early days of our Galaxy as they do now. As outlined by Larson [14], this evidence finds its natural explanation in a time dependent IMF with a bias towards massive stars in the early Universe. Such a varying IMF is favoured for other reasons as well. For example, the gas of galaxy clusters is very hot and contains a large mass of heavy elements, which can not be explained from the proportionally few massive stars of the present day IMF. Also the observed strong evolution of the cosmic luminosity density with redshift is easier to explain with a topheavy IMF. And microlensing experiments indicate that a significant fraction of dark matter is in the form of stellar remnants, which can only be produced from massive progenitors. See Larson's article 14] for a more detailed explanation and references. 


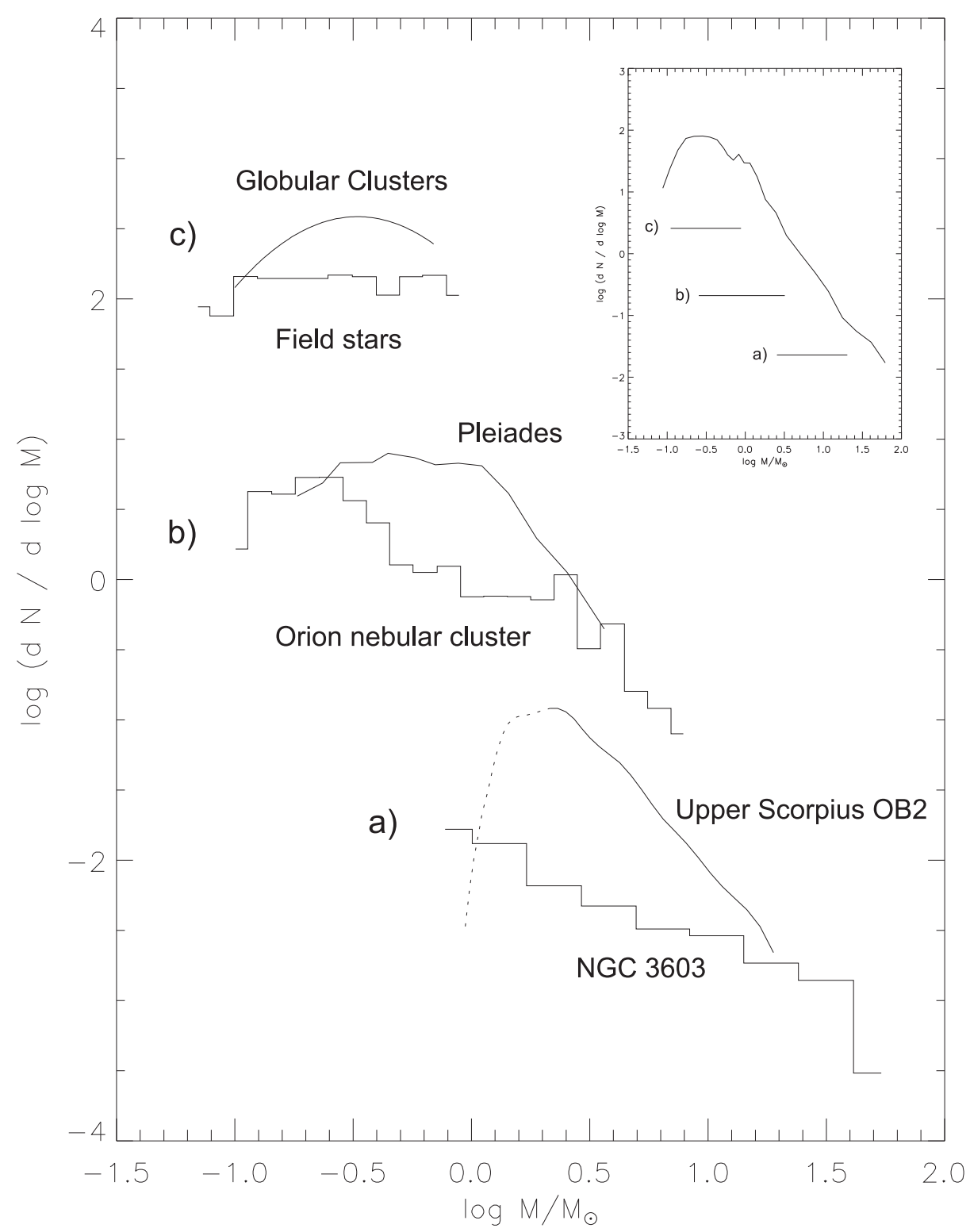

Fig. 1. Evidence for IMF variations: This figure shows a comparison of the IMF of several selected Galactic regions. Variations of the IMF are evident for all stellar masses: a) The exponent of the IMF differs by more than one in the high mass star forming regions NGC 3603 [4] and Upper Scorpius OB2 [2]. b) Compared to the Pleiades [26], the IMF of the Orion nebular cluster [10] is deficit in stars with masses between 0.5 - $1.25 M_{\odot}$. c) The low-mass IMF in globular clusters is fitted best by a log-normal distribution [28], but the field stars follow a power law in the same mass interval [30]. The small inset shows the field star IMF of Scalo [37] for comparison. 


\section{Starbursts and Extragalactic Star Forming Regions}

\subsection{Starburst Galaxies}

Starburst galaxies are another extreme environment for star formation. Their star formation rate is several magnitudes larger than in our own Galaxy. So if the thermal Jeans mass is of any importance for a characteristic stellar mass, the heating from the unusually strong star formation should give preference to more massive stars.

The starburst galaxy M 82 has been the prime example for a top heavy IMF for 20 years [31. As the closest starburst galaxy with a distance of only 3.2 Mpc, no other starburst galaxy has been observed in such detail. The appearance of this galaxy is dominated by its nuclear starburst, which has probably been induced by a close encounter with M 81 about $100 \mathrm{Myr}$ ago. The basic properties of the nuclear starburst are 24]: Bolometric luminosity $>5.0 \times 10^{10} L_{\odot}$, absolute $\mathrm{K}$ magnitude $<-22.5$, ionising radiation $L_{L y c}>10^{54}$ photons $/ \mathrm{s}$, total mass in the starburst $<2.5 \times 10^{8} M_{\odot}, \mathrm{CO}$ index $>0.21$ and supernova rate $\approx 0.1$ $y r^{-1}$. Rieke et al. 31, 32 have pioneered the detailed modelling of this starburst galaxy, and have come repeatedly to the conclusion that stars with masses below a few $M_{\odot}$ form much less often in M 82 than in the solar neighbourhood. The argument for a top heavy IMF is basically that the infrared brightness - tracing the bolometric luminosity — is too high for the (dynamically measured) stellar mass, if one assumes a IMF similar to the field star IMF of Scalo [37]. The ionising radiation, the $\mathrm{CO}$ index and the supernova rate provide the necessary constraints for the age of the stellar population.

Although recent modelling [36.,6] with a more complex spatial structure and temporal evolution can not exclude a Salpeter power-law IMF extending down to subsolar masses, the starburst galaxy M 82 remains one of the most cited examples for IMF variations.

\subsection{Extragalactic Super Star Clusters}

The same technique can also be applied to extragalactic super star clusters. Spectral diagnostics, such as hydrogen emission lines and stellar absorption features, enable us to date the starburst. Dynamical mass estimates together with infrared observations provide a reliable mass-to-light ratio, and these together constrain the shape of the IMF. Compared with starburst galaxies, individual super star clusters offer the big advantage of a simple spatial (only one cluster) and temporal (only one burst) structure. Nevertheless these clusters can be looked upon as the building blocks of starburst galaxies, and should thus trace the IMF for rather extreme environmental conditions, too. However, the IMF in starburst clusters is not necessarily biased towards massive stars.

The first super star clusters with dynamical mass estimates were NGC 1569A and NGC 1705-1 [12]. With a mass of $1.1 \times 10^{6} M_{\odot}\left(\mathrm{NGC} \mathrm{1569A)}\right.$ and $2.7 \times 10^{5}$ $M_{\odot}$ (NGC 1705-1), these clusters are both gravitationally bound and could 
evolve into globular clusters $\sqrt{12}$. However, recent evolutionary synthesis modelling of the mass-to-light ratio [39] revealed that the IMF must be very different in the two regions. While the IMF in NGC 1596A is steep (slope $\approx-1.5$ ) and extends to below $0.1 M_{\odot}$, the IMF in NGC 1705-1 is either flat (slope $>-1$ ) or truncated at a lower mass limit of $1-3 M_{\odot}$.

A similar conclusion has been found for the Antennae 25. The new generation of infrared spectrometers has allowed the dynamical mass determination of young embedded super star clusters for the first time. The authors 25 have compared each mass measurement with the results with the stellar synthesis model Starburst99 [15], that fit best the observed NIR-luminosity, Br $\gamma$, and the $\mathrm{CO}$ and $\mathrm{Ca}$ absorption features. The measurements in two of the observed clusters are consistent with a Salpeter IMF extending to subsolar masses. However, in their cluster \#2 [43], such a power law IMF with a slope of -1.35 would include all the dynamical mass $\left(\approx 1.6 \times 10^{6} M_{\odot}\right)$ in stars more massive than 1 $M_{\odot}$. Therefore no mass is left for subsolar mass stars. This cluster seem to have formed proportionally more massive stars.

In conclusion, some of the measured mass-to-light ratios in super star clusters support the theoretical arguments for a top heavy IMF in starbursts, and thus systematic variations in the IMF, but the same kind of measurements also point to random variations in the IMF from cluster to cluster, even those in similar environments.

\subsection{Extragalactic Star Formation Complexes}

Unfortunately there are only a few dynamical mass measurements for star clusters. Statistics on IMF variations for a larger number of extragalactic star forming regions has to rely on integrated photometric and spectroscopic properties.

A recent example for such a statistical approach is [34], who have included 105 extragalactic star formation complexes for which UBVR photometry, Lyman continuum flux, metallicity and extinction measurements were available. These properties trace essentially the high mass stellar content, and the results are restricted to the IMF for stars more massive than $10 M_{\odot}$. The models include the slope of the IMF, the maximum stellar mass and the age of the cluster. The star formation history is assumed either as a delta burst or as continuous. The average slope of the IMF was found to be -1.42. The standard deviation in the measured distribution is 0.91 , and the authors estimate the standard error of their method to be 0.51. Therefore the measured distribution of slopes is meaningful 34]. Because the total number of analysed star formation complexes is rather large, the formal probability of measuring such a large standard deviation is basically negligible $\left(<10^{-10}\right)$, if one assumes a universal IMF.

Another investigation [23] has concentrated on 17 star formation regions in a sample of blue compact and irregular galaxies, using the integrated properties from UV, optical, FIR and radio measurements. This analysis uses the equivalent widths of $\mathrm{H} \beta$, Si IV, and Ca IV to constrain the relative number of O3-O8 stars to B0-B3 stars, and thus the slope of the high mass IMF for stars more massive than $10 M_{\odot}$. The evolutionary tracks of starburst regions with three modelled IMF 
slopes of $0.0,-1.35,-2.0$ are clearly separated in a $\mathrm{W}(\mathrm{H} \beta)$ over $\mathrm{W}(\mathrm{Si}$ IV $) / \mathrm{W}(\mathrm{C}$ IV $)$ diagram. The derived slope for the starburst regions varies between 0 (3 regions), -1.35 (8 regions) and -2.0 (5 regions). Despite the variation in the derived slopes of the IMF, the authors interpret their result as in supporting a universal IMF, specifically because they see no trend with metallicity 23. However, the scatter in the diagram is much larger than the average error bars, and I would interpret the same diagram as evidence for a varying IMF. In addition, the authors also report that objects showing flatter IMFs are always small compact star forming regions, that four of the galaxies with optical continuum dominated by a previous generation of stars have IMF slopes close to -2 , and that a previous burst of star formation could have hampered the formation of lower mass stars.

\section{IMF Variations in the Galaxy}

A direct measurement of the IMF from star counts is still restricted to our Galaxy and the most nearby galaxies. While we can only compare the relative number of high- to low-mass stars or constrain the slope of the high-mass IMF in the early Universe and in extragalactic starburst regions, we are able to trace the IMF down to substellar masses in nearby star forming regions. Figure 1 shows a comparison of several IMFs measured in the Galaxy. We find variations in the slope and/or the shape of the IMF for all mass ranges.

\subsection{The Most Massive Star Forming Regions}

The most massive stars found in the Galaxy have about $100 M_{\odot}$. Most of these stars have been found in either very compact star clusters or rather loose OB associations. The observations of these regions have improved significantly in the last few years with the HIPPARCOS mission, the Hubble Space Telescope (HST) and adaptive optics assisted telescopes.

For example, HIPPARCOS has revealed 178 new members of the Upper Scorpius OB2 association [2]. Only 91 stars were previously known in this association. Figure 1 shows the preliminary IMF. With a slope of $\approx-1.9$, this IMF resembles very well Scalo's [37] field star IMF. I have compared this high-mass IMF with the IMF in NGC 3603, the most massive visible HII region in our Galaxy [4. Adaptive optics assisted observations have revealed more than 800 stars in the central parsec of this cluster. Although the IMF shows no turnover or truncation down to $<1 M_{\odot}$, its slope is only $\approx-0.7$ for stars with masses between 3 and 30 $M_{\odot}$. A very similar result was found with the help of HST in the Arches cluster near the Galactic centre [5]. The slope of the IMF for stars more massive than $10 M_{\odot}$ is $\approx-0.65$, much shallower than the reference field star IMF, too. The Arches cluster and NGC 3603 are two of the most massive young clusters in the Galaxy, and each cluster has a few $1000 M_{\odot}$ in O-stars. Therefore the difference between the field star IMF and the IMF in NGC 3603 and the Arches cluster can not be explained by small number statistics. Such an argument is often used to invalidate the evidence for IMF variations from star counts [13]. The finding 
in these two clusters supports the hypothesis that the IMF in starburst regions is biased towards massive stars. However, the deficit of low-mass stars is caused by a shallow IMF, and does not result from a truncated IMF, which has been indicated in M 82 32.

\subsection{Associations and Open Clusters}

The discussion about a universal IMF was revived by Scalo 38 in 1997, at a time, when the majority of the participants of a conference on the stellar initial mass function was arguing for a universal IMF [7]. He made his case for IMF variations basically from a statistical interpretation of the measured slopes of the IMF in Galactic and Magellanic cloud associations and open clusters. Scalo plotted the slope of the IMF of 61 clusters against the average logarithmic stellar mass considered in the measurements. Although the typical error in the slopes is $0.1-0.4$, the spread in the diagram above $\approx 1 M_{\odot}$ is so large that the author saw no basis for adopting some average value.

A serious argument against such evidence for IMF variations is that the results for different clusters have been obtained from different authors using different techniques. Therefore the IMF variations may have been mimicked by systematic errors, which have not been addressed carefully enough. I have thus repeated a similar $\chi^{2}$ analysis for this conference to estimate how large the systematic errors would have to be, if the IMF were to be universal. My compilation includes the slopes of the IMFs for 51 clusters with intermediate- to high-mass star formation from 7 publications. The publications have been selected such that each paper includes more than 4 clusters, so that I could carry out statistics for each author and technique separately. Table 11 summerises the IMF properties of the different publications. Clusters with suspicious IMF measurements (for example NGC 436 in [29], which has probably undergone dynamical evolution), and results without error estimates (for example NGC 7235 in [21]) or with a different mass range (for example NGC 376 and N 24 in [9]) have not been included in the statistics. For the ease of interpretation, I have converted the reduced $\chi^{2}$ values into a significance level [1], which is the probability of measuring such a high or higher $\chi^{2}$, if the IMF would have a universal slope equal to the weighted mean slope in this subsample. Even at a significance level of only $5 \%$, we have to reject the hypothesis of a universal IMF in 5 of 7 cases. The statistics for the whole sample is even worse. The standard deviation in the slope distribution is 1.9 times the mean error in the measurements of the slopes. The likelihood for such a large discrepancy is basically negligible $\left(<10^{-10}\right)$ for a universal IMF. If the IMF is indeed universal, the authors must have underestimated their errors by a factor of two. The systematic errors would have to be even larger if we include the field stars of the Magellanic clouds, which show a particularly steep IMF with a slope of $\approx-3$ to -4 [22].

A very intriguing example with very different IMFs at intermediate stellar masses are the Pleiades and the Orion nebular cluster. These clusters have been the target of some of the most careful recent measurements of the IMF [26,10]. The IMFs of these two clusters are also displayed in figure $\mathbb{1}$. In contrast to the 
Table 1. IMF variations in 57 clusters with intermediate- to high-mass star formation

\begin{tabular}{lllllll}
\hline $\begin{array}{l}\text { Ref. } \\
\text { of } \\
\text { clusters }\end{array}$ & $\begin{array}{l}\text { Number } \\
\text { slope }\end{array}$ & $\begin{array}{l}\text { Weighted } \\
\text { in slopes }\end{array}$ & $\begin{array}{l}\text { Standard } \\
\text { deviation } \\
\text { in }\end{array}$ & $\begin{array}{l}\text { Mean error } \\
\text { in slope } \\
\text { measurements }\end{array}$ & $\begin{array}{l}\text { Reduced } \\
\chi^{2}\end{array}$ & Significance \\
\hline 40 & 4 & 1.43 & 0.34 & 0.15 & 5.15 & $0.15 \%$ \\
\hline 33 & 5 & 1.27 & 0.80 & 0.33 & 14.82 & $\approx 0^{\mathrm{a}}$ \\
\hline 27 & 6 & 1.22 & 0.31 & 0.30 & 2.92 & $1.2 \%$ \\
\hline 21 & 12 & 1.12 & 0.44 & 0.34 & 1.78 & $5.3 \%$ \\
\hline 22 & 5 & 1.27 & 0.18 & 0.16 & 1.64 & $16.2 \%$ \\
\hline 9 & 12 & 1.92 & 0.54 & 0.34 & 3.22 & $0.021 \%$ \\
\hline 29 & 7 & 1.42 & 0.26 & 0.15 & 18.08 & $\approx 0^{\mathrm{a}}$ \\
\hline all & 51 & 1.52 & 0.53 & 0.28 & 6.35 & $\approx 0^{\mathrm{a}}$
\end{tabular}

a The significance level is $<10^{-10}$.

IMF of the Pleiades, which follows the field star IMF, the IMF in Orion shows a regime of "missing" stellar masses between $0.5-1.25 M_{\odot}$. Other examples are NGC 6231 and NGC 2264. The IMFs in these clusters have been measured in both cases with the same technique - UBVRI and $\mathrm{H} \alpha$ photometry - and by the same authors 41 42], therefore minimising systematic variations. Nevertheless, the IMF in NGC 2264 rises continously down to below $0.6 M_{\odot}$, whereas the IMF decreases abruptly below $2.5 M_{\odot}$ in NGC 6231 .

\subsection{Low-Mass Stars in Young Clusters, Globular Clusters and the Field}

If we assume a Salpeter 35] power-law IMF with a slope of -1.35 extending down to a lower mass limit of $0.1 M_{\odot}, 96 \%$ of all stars would have a mass smaller than $1 M_{\odot}$, and $55 \%$ of the stellar mass would be included in stars less massive than the sun. However, these low-mass stars are very faint, and many such stars have not been detected before the large ground based infrared surveys and HST. These observations have also revealed IMF variations in many regions.

To illustrate these variations, I will again compare different regions with the field star population. Figure 1 shows a recent measurement [30] of the field star IMF for low-mass stars. It was derived from the Deep Near-Infrared Survey (DENIS) and the 2 Micron All-Sky Survey (2MASS). In the $0.1-1 M_{\odot}$ mass range, this IMF can be represented by a power-law mass function with a slope of $\approx 0.1$. The statistical uncertainties are $\approx 0.13$. A similar IMF has been observed in the young star forming regions IC 348, $\rho$ Ophiuchi, and the Trapezium 16, 17, 18. Their IMF is flat or slowly rising - slope $\gtrsim 0$ - from the brown dwarf regime to $0.6-1 M_{\odot}$, where it rolls over to a power-law with a slope of $\approx-1.7$. It is important to note that the IMF is not log-normal 18 .

In contrast, recent HST observations indicate that the IMF of globular clusters can be described by a log-normal function with a peak near $0.3 M_{\odot}$ [28]. 
Figure 1 shows the best fit for a sample of 12 globular clusters. The authors [28] explicitly exclude a single power-law IMF in the $0.1-0.6 M_{\odot}$ range. Therefore the IMF of globular clusters seems to be fundamentally different from the IMF in young star forming regions and the field.

A meaningful interpretation of the IMF measurements for masses below the hydrogen burning limit is increasingly more difficult, because the available data are sparse. However, observations of young star forming regions indicate IMF variations even in the mass regime of brown dwarfs. The Taurus star forming region, for example, is significantly deficient in objects below $0.1 M_{\odot}$ compared to the Trapezium [19]. If the IMF of both objects is normalised by the number of stars between $0.1-1 M_{\odot}$, then $\approx 13$ brown dwarfs with masses $>0.02 M_{\odot}$ are found in the Trapezium, but only one in Taurus.

\section{Conclusions}

Variations of the stellar initial mass function have been reported for all masses and in a large variety of stellar populations. We find evidence for IMF variations in the early Universe, in starburst galaxies and extragalactic star forming regions, in Galactic star clusters and associations, and in the field star population. Neither the shape nor the slope and the characteristic mass of the IMF seem to be excluded from these variations. There is some indication that the IMF is systematically biased towards more massive stars in the early Universe and in starbursts. However, I see no obvious systematic trend in those regions where the IMF could be constructed from direct star counts. If the IMF is indeed universal, it will be very difficult to prove this postulation empirically, because every individual measurement of a different IMF has to be invalidated. Only a consistent theory of star formation, with clear and testable predictions, will finally convince in the scientific world.

\section{References}

1. T.C. Beers: 'Observational Contraints on the Nature of the First Stars - Final Comments'. In: The First Stars, MPA/ESO workshop, Garching, Germany, August 4-6, 1999, ed. by A. Weiss, T.G. Abel, V. Hill (Springer, Heidelberg, 2000), pp. $336-342$

2. A.G.A. Brown: 'The Initial Mass Function in Nearby OB Associations'. In: The Stellar Initial Mass Function, 38th Herstmonceux Conference, Cambridge, UK, July 14-18, 1997, ed. by G. Gilmore, D. Howell (ASP, San Francisco, 1998), ASP Conference Series, 142 , pp. 45-59

3. C. Chiosi: 'Evolution of Pop III Stars'. In: The First Stars, MPA/ESO workshop, Garching, Germany, August 4-6, 1999, ed. by A. Weiss, T.G. Abel, V. Hill (Springer, Heidelberg, 2000), pp. 95-110

4. F. Eisenhauer, A. Quirrenbach, H. Zinnecker, R. Genzel: ApJ 498, 278 (1998)

5. D.F. Figer, S.S. Kim, M. Morris, E. Serabyn, R.M. Rich, I.S. McLean: ApJ 525, 750 (1999) 
6. N. Förster-Schreiber: Near-infrared imaging spectroscopy and mid-infrared spectroscopy of M82: revealing the nature of star formation activity in the archetypal starburst galaxy. Ph.D. thesis, Ludwig-Maximilians-Universität, München (1998)

7. G. Gilmore, D. Howell (eds.): The Stellar Initial Mass Function, 38th Herstmonceux Conference, Cambridge, UK, July 14-18, 1997 (ASP, San Francisco, 1998), ASP Conference Series, 142

8. G. Gilmore: these proceedings

9. R.J. Hill, B.F. Madore, W.L. Freedman: ApJ 429, 192 (1994)

10. L.A. Hillenbrand: AJ 113, 1733 (1997)

11. ISO Information Centre: Statistical methods, Handbook on international standards for statistical methods (International Standards Organization, Geneva, 1979)

12. L.C. Ho, A.V. Filippenko: ApJ 466, L83 (1996)

13. P. Kroupa: MNRAS, accepted (2000)

14. R.B. Larson: MNRAS 301, 569 (1998)

15. C. Leitherer, D. Schaerer, J.D. Goldader, R.M.G. Delgado, C. Robert, D.F. Kune, D.F. de Mello, D. Devost, T.M. Heckman: ApJS 123, 3 (1999)

16. K.L. Luhman, G.H. Rieke, C.J. Lada, E.A. Lada: ApJ 508, 346 (1998)

17. K.L. Luhman, G.H. Rieke: ApJ 525, 440 (1999)

18. K.L. Luhman, G.H. Rieke, E.T. Young, A.S. Cotera, H. Chen, M.J. Rieke, H. Schneider, R.I. Thompson: ApJ 540, 1016 (2000)

19. K.L. Luhman: ApJ 544, 1044 (2000)

20. A. Martinelli, F. Matteucci: A\&A 353, 269 (2000)

21. P. Massey, K.E. Johnson, K. DeGioia-Eastwood: ApJ 454, 151 (1995)

22. P. Massey, C.C. Lang, K. Degioia-Eastwood, C. Garmany: ApJ 438, 188 (1995)

23. J.M. Mas-Hesse, D. Kunth: A\&A 349, 765 (1999)

24. K.K. McLeod, G.H. Rieke, M.J. Rieke, D.M. Kelly: ApJ 412, 111 (1993)

25. S. Mengel: these proceedings

26. H. Meusinger, E. Schilbach, J. Souchay: A\&A 312, 833 (1996)

27. M.S. Oey: ApJ 465, 231 (1996)

28. F. Paresce, G. De Marchi: ApJ 534, 870 (2000)

29. R.L. Phelps, K.A. Janes: AJ 106, 1870 (1993)

30. I.N. Reid, J.D. Kirkpatrick, J. Liebert, A. Burrows, J.E. Gizis, A. Burgasser, C.C. Dahn, D. Monet, R. Cutri, C.A. Beichman, M. Skrutskie: ApJ 521, 613 (1999)

31. G.H. Rieke, M.J. Lebofsky, R.I. Thompson, F.J. Low, A.T. Tokunaga: ApJ 238, $24(1980)$

32. G.H. Rieke, K. Loken, M.J. Rieke, P. Tamblyn: ApJ 412, 99 (1993)

33. R. Sagar, W.K. Griffiths: MNRAS 299, 777 (1998)

34. F. Sakhibov, M. Smirnov: A\&A 354, 802 (2000)

35. E.E. Salpeter: ApJ 121, 161 (1955)

36. S. Satyapal, D.M. Watson, J.L. Pipher, W.J. Forrest, M.A. Greenhouse, H.A. Smith, J. Fischer, C.E. Woodward: ApJ bf 483, 148 (1997)

37. J.M. Scalo: Fundamentals of Cosmic Physics 11, 1 (1986)

38. J.M. Scalo: 'The IMF Revisited: A Case for Variations'. In: The Stellar Initial Mass Function, 38th Herstmonceux Conference, Cambridge, UK, July 14-18, 1997, ed. by G. Gilmore, D. Howell (ASP, San Francisco, 1998), ASP Conference Series, 142, pp. 201-236

39. A. Sternberg: ApJ 506, 721 (1998)

40. A. Subramaniam, R. Sagar: AJ 117, 937 (1999)

41. H. Sung, M.S. Bessel, S.-W. Lee: AJ 114, 2644 (1997)

42. H. Sung, M.S. Bessel, S.-W. Lee: AJ 115, 734 (1998)

43. B.C. Whitmore, Q. Zhang, C. Leitherer, S.M. Fall, F. Schweizer, B.W. Miller: AJ 118, 1551 (1999) 